\title{
Postpartum haemorrhage: various method of management in arural tertiary care hospital
}

\author{
Mahendra G. ${ }^{1}$, Ramesh Babu ${ }^{2}$, Anshu Kumari ${ }^{3}$, Pukale R.S. ${ }^{4}$ \\ ${ }^{1}$ Dr Mahendra G., Associate Professor, ${ }^{2}$ Dr Ramesh Babu, Associate Professor, ${ }^{3}$ Dr Anshu Kumari, Junior Resident, \\ ${ }^{4}$ Prof Dr Ravindra S. Pukale, Professor and Head of the Department; all authors are affiliated with Department of \\ Obstetrics and Gynaecology, Adichunchanagiri Institute of Medical Sciences, Nagamangala Taluk, Mandya District, \\ Karnataka, India.
}

Corresponding Author: Dr Anshu Kumari, Department of Obstetrics and Gynaecology, Adichunchanagiri Institute of Medical Sciences, Nagamangala Taluk, Mandya District, Karnataka, India, E-mail: anshukri1407@gmail.com

\begin{abstract}
Introduction: Post-partum haemorrhage is defined as blood loss from the genital tract, exceeding 500ml within 24hours of vaginal delivery and $1000 \mathrm{ml}$ during caesarean section. It is also now a day defined as any blood loss that has a potential to produce or produces hemodynamic instability. Massive obstetric haemorrhage is a major cause of maternal mortality and morbidity worldwide. Aims and Objectives: Objective of the study is to evaluate the most common etiology and method of management of Postpartum Haemorrhage in a rural tertiary care hospital of SAH \&RC. Materials and Methods: A prospective study was done from June 2017 to June 2018 in Department of Obstetrics and Gynaecology at Adichunchanagiri Institute of Medical sciences, Bellur, Karnataka, India. The study included all cases of normal vaginal deliveries, assisted vaginal deliveries and Caesarean section. Result: A total of 1877women were delivered. Out of which 923 (49.17\%) women delivered vaginally and 954 (50.82\%) women delivered through cesarean section. Out of 1877 delivered women, 69 (3.67\%) developed PPH. out of 69(3.79\%), 66(95.65\%) had atonic PPH and $3(4.34 \%)$ had traumatic PPH. out of 66 atonic PPH cases 54(81.81\%) managed medically. Other modalities were B lynch suture $7(10.6 \%)$, Hayman suture 2(3.03\%), suction cannula 1(1.51\%), internal iliac artery ligation $1(1.51 \%)$, peripartum hysterectomy $1(1.51 \%)$. Most of the cases have been managed medically. Conclusion: In an era with availability of excellent uterotonics and active management of third stage of labor even today PPH stands first as the cause of maternal mortality and morbidity. This study highlights the existing variable practices for management of PPH. Haemorrhage associated mortality can be prevented by critical judgment and early intervention.
\end{abstract}

Keywords: Post-partumhaemorrhage, Atonic PPH, Traumatic PPH.

\section{Introduction}

Post-partum haemorrhage is the leading cause of maternal morbidity and mortality and accounts for one quarter of cases of maternal mortality worldwide [1]. It is among the manageable causes of maternal mortality that if managed properly can prevent most of the cases of maternal deaths.

According to WHO, obstetric haemorrhage causes 127000 deaths annually worldwide and is the leading cause of maternal mortality [2]. Post-partum haemorrhage is defined as blood loss from the genital tract, exceeding $500 \mathrm{ml}$ within $24 \mathrm{hrs}$ of vaginal delivery and $1000 \mathrm{ml}$ during caesarean section [3].

Manuscript received: $28^{\text {th }}$ March 2019

Reviewed: $07^{\text {th }}$ April 2019

Author Corrected: $12^{\text {th }}$ April 2019

Accepted for Publication: $18^{\text {th }}$ April 2019
It is also now a day defined as any blood loss that has a potential to produce or produces hemodynamic instability. Massive obstetric haemorrhage is a major cause of maternal mortality and morbidity worldwide. It is not only life-threatening situation but also an obstetrician'snightmares [4]. Many national and international studies show that uterine atony is the commonest cause of PPH followed by trauma to genital tract, adherent placenta, uterine angle extension and retained placenta [5]. Primary (immediate) PPH occurs within the first 24 hours after delivery. Approximately $70 \%$ ofimmediate PPH are due to uterine atony. Atony of the uterus is defined as the failure of the uterus to contract adequately after the child is born [6]. Uterine atony is the most common cause of $\mathrm{PPH}$, in about 75$90 \%$ of cases. 


\section{Original Research Article}

Other causes include placenta previa, accreta, lower genital tract laceration, coagulopathy, uterine inversion and ruptured uterus [7]. Secondary (late) PPH occurs between 24 hours after delivery of the infant and 6 weeks postpartum. Most late PPH is due to retained products of conception, infection, or both [6]. Generally, PPH requires early recognition of its cause, immediate control of the bleeding source by medical, mechanical, invasive-non-surgical and surgical procedures, rapid stabilization of the mother's condition, and a multidisciplinary approach [8]. PPH can be minor (500-1000 ml) or major (more than 1000 $\mathrm{ml})$, major can be divided into moderate (1000 -2000 $\mathrm{ml}$ ) and severe (more than $2000 \mathrm{ml}$ ) [9].

Oxytocin, syntometrine, ergometrine, PGF2 alpha and misoprostol are different medical preparations used as uterotonics for prophylaxis and therapeutic management of PPH. The two main aspects of management of PPH are resuscitation and identification/management of underlying cause. Interventions like application of compression sutures, internal iliac artery ligation, uterine artery embolization and hysterectomy are other life saving measures.

\section{Aims and Objective}

1) To find out the incidence of post postpartum haemorrhage.

2) To analyze various modalities used in the management of postpartum haemorrhage.

3) To study maternal outcome in PPH.

\section{Materials and Methods}

Study type- Prospective study

Study period-June 2017 to June 2018 in Department of Obstetrics and Gynaecology at Adichunchanagiri Institute of Medical sciences.

Study area- Tertiary care hospital, Adichunchangiri Institute of Medical Sciences, B.G Nagara, Nagamangala Taluk, Mandya Distract, Karnataka

Inclusion criteria: This study included all patients admitted for delivery \& ending up in PPH or presenting with $\mathrm{PPH}$ in casualty or referred from outside as $\mathrm{PPH}$.

Exclusion criteria: Patients with history of coagulation disorder \& patients who were taking heparin \&warfarin.

Sample collection- The study included all cases of normal vaginal deliveries, assisted vaginal deliveries and Caesarean section over astudy period of June 2017 to June 2018. For calculation of frequencies, the total number of deliveries in the setting during study period was used. Diagnosis of PPH was made clinically based on findings of pelvic examination, condition of uterus and amount of bleeding. Maternal condition was assessed and managed according to established hospital protocols which included both medicaland surgical interventions.All maternal complications were noted and recorded in predesigned proforma.Ethical consideration \& permission obtained fromInstitutional Ethical Committee

\section{Results}

Duringstudy period ofone year, total of 1877 women were delivered. Out of which $923(49.17 \%)$ women delivered vaginally and $954(50.82 \%)$ women delivered through caesarean section.Out of1877 delivered women, 69 (3.67\%) developed PPH. Out of 69 PPH cases 3, were referred cases.

\begin{tabular}{|c|c|}
\hline Total No of Deliveries & 1877 \\
\hline Number of PPH Cases & 69 \\
\hline
\end{tabular}

Out of 69 who developed PPH, 40 (57.97\%) were primipara and 29 (42.02\%) were multipara.

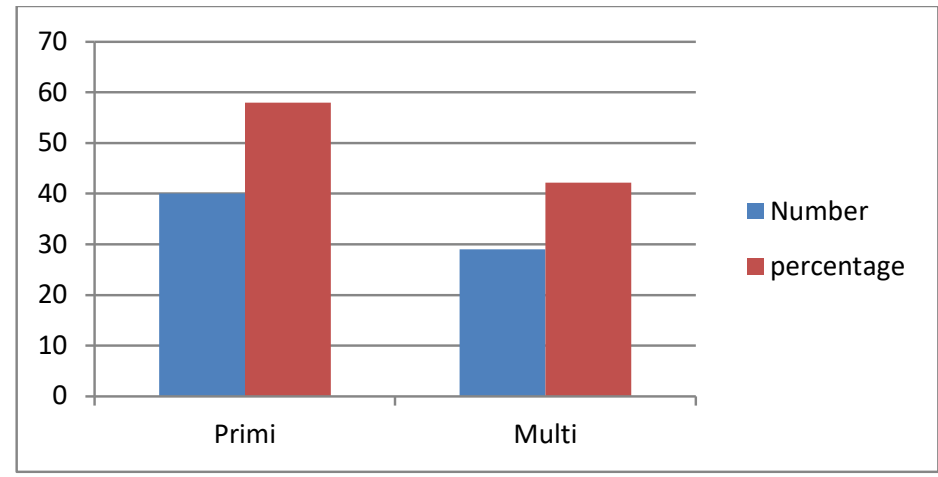


Table-1: Presence ofhigh-risk factor

\begin{tabular}{|c|c|c|}
\hline High risk factors & Numbers & Percentage \\
\hline No any factor & 49 & 71.01 \\
\hline Anemia & 3 & 4.35 \\
\hline Preeclampsia/ eclampsia & 8 & 11.59 \\
\hline Twins/ polyhydramnios & 3 & 4.35 \\
\hline Gestational diabetes mellitus & 6 & 8.69 \\
\hline
\end{tabular}

In $71 \%$ of PPH cases there was no identifiable risk factor

Table-2: Incidence of PPH in different modeof delivery

\begin{tabular}{|c|c|c|}
\hline Title & Numbers & Percentage \\
\hline PPH in vaginal delivery & 45 & $4.87 \%$ \\
\hline PPH in instrumental vaginal delivery & 3 & $5.08 \%$ \\
\hline PPH in caesarean section & 21 & $2.20 \%$ \\
\hline
\end{tabular}

This study found that maximum incidence of $\mathrm{PPH}$ is associated with instrumental vaginal delivery (5.08\%) followed by vaginal $(4.87 \%)$, and caesarean section $(2.20 \%)$

Table-3: Cause of PPH

\begin{tabular}{|c|c|c|}
\hline PPH cause & Number & Percentage \\
\hline Uterine atony & 66 & $95.65 \%$ \\
\hline Traumatic & 3 & $4.34 \%$ \\
\hline
\end{tabular}

Out of 69, 66(95.65\%) had atonic PPH and 3(4.34\%) had traumatic PPH. Main cause of PPH in this study is uterine atony i.e. $95.65 \%$ and 2 nd common cause is traumatic i.e. $4.34 \%$.

Out of 66 atonic PPH cases 54(81.81\%) managed medically, B lynch suture 7(10.6\%), Hayman suture 2(3.03\%), suction cannula 1(1.51\%), internal iliac artery ligation 1(1.51\%), peripartum hysterectomy 1(1.51\%). Most of the cases have been managed medically. In this study, out of69 who developed PPH there was 1 maternal mortality which was referred case.

In this studymedical management was done by giving 1)oxytocin10 IU IM or 20-40 IU in 1 liter ofnormal saline at 60 drops per minute, and continue oxytocin (20 IU in 1 litre of iv fluids at 40 drops per minute) until haemorrhage stops, 2) Ergometrine $0.2 \mathrm{mg}$ im or can be given slowly iv may be repeated as required at interval of 2-4 hours, 3) PGF2 $\alpha$ or carboprost $0.25 \mathrm{mg}$ dose can be repeated every 15 minutes for a total dose of $2 \mathrm{mg}$ (maximum of 8 doses), 4)PGE1 or misoprostol dose vary from $400 \mu \mathrm{g}$ to maximum of $1200 \mu \mathrm{g}$.

Table-4: Management protocol of PPH

\begin{tabular}{|c|c|c|}
\hline Methods of management & Number & Percentage (\%) \\
\hline Medical management & 54 & $81.81 \%$ \\
\hline B Lynch suture & 7 & $10.60 \%$ \\
\hline Hayman suture & 2 & $3.03 \%$ \\
\hline Suction cannula & 1 & $1.51 \%$ \\
\hline Internal iliac artery ligation & 1 & $1.51 \%$ \\
\hline Peripartum hysterectomy & 1 & $1.51 \%$ \\
\hline
\end{tabular}

\section{Discussion}

Postpartum hemorrhage is the leading cause of morbidity and mortality among pregnant ladies throughout the world causing 140,000 deaths each year globally, this corresponds to the one women dying in every 4 minutes and it is the 5 th most common cause of maternal mortalitythroughout the world [10,11-15]. Postpartum hemorrhage is a leading cause of 


\section{Original Research Article}

maternal mortality. The place of delivery and severity of hemorrhage determine the outcome. Commonest mode of delivery which was found in present study was C-sectionfollowed by spontaneous vaginal delivery and instrumental vaginal delivery.If the woman has developed PPH following delivery in a health facility, the immediate medical and surgical interventions are possible. It is not so, when woman delivers at home or in a small hospital ill equipped with facilities to manage obstetric emergencies, Diagnosis of PPH and decision to transfer to hospital or tertiary care Centre is very crucial. Home deliveries and deliveries in small facilities have negative influence on the outcome.Most important and major finding in our study was that the most common cause ofpostpartum hemorrhage was uterine atony, which is loss of tone in the uterine musculature. Data support the routine use of active management of the third stage of labor (AMTSL) by all skilled birth attendants, regardless of where they practice; AMTSL reduces the incidence of PPH, the quantity of blood loss, and the need for blood transfusion, and thus should be included in any program of intervention aimed at reducing death from PPH [16]. The usual components of AMTSL include: 1) Administration of oxytocin (the preferred storage of oxytocin is refrigerationbut it may be stored at temperatures up to $30^{\circ} \mathrm{C}$ for upto 3 months without significant loss of potency) or another uterotonicdrug within 1 minute after birth of the infant. 2) Controlled cord traction.3) Uterine massage after delivery of the placenta.The Bristol [17] and Hinchingbrooke [18] studies compared active versus expectant (physiologic) management of the third stage oflabor. Both studies clearly demonstrated that, when active management was applied, the incidence of PPH was significantly lower $(5.9 \%$ with AMTSL vs $17.9 \%$ with expectant management [17]; and6.8\% with AMTSL vs 16.5\% without [18]. FIGO promotes the routine use of AMTSL as the best, evidence-based approach for the prevention of PPH and emphasizes that every effort should be made to ensure that AMTSL is used at every vaginal birth where there is a skilled birth attendant. However, FIGO recognizes that there may be circumstances where the accessibility or the supply of uterotonics may be sporadic owing to interruptions in the supply chain, or it may be nonexistent in a country because it is not part of the approved list of essential medicines or included in the national guidelines/protocols. It is in this context that the birth attendant must know how to provide safe care (physiologic management) to prevent PPH in the absence of uterotonic drugs [6]. Most maternal deaths due to PPH occur in low income countries in settings (both hospital and community) where there are no birth attendants or where birth attendants lack the necessary skills or equipment to prevent and manage PPH and shock. The Millennium Development Goal of reducing the maternal mortality ratio by $75 \%$ by 2015 will remain beyond our reach unless we prioritize the prevention and treatment of PPH in low-resource areas [19].

Table-5: Incidence of PPH in different study

\begin{tabular}{|c|c|}
\hline Study & Incidence (\%) \\
\hline Present study & $3.67 \%$ \\
\hline Tasneem F et al [20] & $3.55 \%$ \\
\hline Edhi et al. [22] & $1.7 \%$ \\
\hline
\end{tabular}

In this study, incidence of PPH was found to be $3.67 \%$, this finding is similar when it is compared with the study conducted by Tasneem F et al [20], in which incidence of PPH was found to be $3.55 \%$. A systematic re-view reported the highest rates of PPH in Africa (27.5\%), and the lowest in Oceania (7.2\%), with an overall rate globally of $10.8 \% .9$ The rate in both Europe and North America was around 13\% [22]. In this study we found bimodal distribution of incidence of PPH in relation to parity i.e. primipara $57.97 \%$ and multipara $42.03 \%$. Reason being different predisposing actors in primigravida like teenage pregnancy, preeclampsia, eclampsia, abruption, anemia, dysfunctional labor, uterine over activity while high parity is the reason in multipara. In $71 \%$ of PPH there is no identifiable risk factor but PPH is not major in this group. We found major PPH in maximum patients with one or more risk factors like anemia, preeclampsia, eclampsia, antepartumhaemorrhage and twins.

Table-6: Incidence of PPH association with different mode of delivery in different study

\begin{tabular}{|c|c|c|c|}
\hline Mode of delivery & Vaginal & Instrumentalvaginal & Caesarean section \\
\hline Present study & $4.87 \%$ & $5.08 \%$ & $2.20 \%$ \\
\hline Solanke et al $\{21\}$ & $1.32 \%$ & $5.26 \%$ & $1.80 \%$ \\
\hline
\end{tabular}

This study observed thatincidence of PPH was maximum with instrumental vaginal delivery (5.08\%), followed by vaginal delivery (4.87) and caesarean section(2.20\%), this findingis similar to the study conducted by Solanke et al[21], in which they observed that maximum incidence of PPH is with instrumental vaginal delivery that is $5.36 \%$, followed by caesarean section $(1.80 \%)$ and vaginal delivery $(1.32 \%)$ 
Original Research Article

Table-7: Incidence of different causes of PPH in different study

\begin{tabular}{|c|c|c|}
\hline Causes & Uterine atony & Traumatic \\
\hline Present study & $95.65 \%$ & $4.34 \%$ \\
\hline Tasneem et al [19] & $86 \%$ & $9.9 \%$ \\
\hline Solanke et al [20] & $79.17 \%$ & $16.67 \%$ \\
\hline
\end{tabular}

In this studymajor cause of PPH was found to be the uterine atonythat is $95.65 \%$, this finding is similar when it is compared with the study conductedby Tasneem et al [19], in which they also found that uterine atony is the major cause of PPH (86\%), whereas study conducted by Solanke et al [20], they found $79.17 \%$ of PPH is due to uterine atony. In international studies uterine atony was the most common cause of $\mathrm{PPH}$, ranging from $50 \%$ to $76 \%$ of cases [23,24].

\section{Conclusion}

In an era with availability of excellent uterotonics and active management of third stage of labor even today PPH stands first as the cause of maternal mortality and morbidity. This study highlights the existing variable practices for management of PPH. The frequency and impact of severe hemorrhage can be effectively reduced by reducing avoidable risk factors, especially those related to obstetric interventions as increased CS rate and induction and augmentation of labour with injudicious use of uterotonics. Other risk factors not amenable to change such as age, ethnic origin, and preexisting medical diseases or bleeding disorders can be minimized byextra vigilance and planned conjoined management.Uterine atony is the most common cause ofpostpartum haemorrhage, so its incidence can be lowered by universal adoption of AMTSL. Institutional deliveries, timely intervention, judicial approach anaesthesia, ICU back up and availability of blood and blood component facilitieswill improve clinical outcome. Avoidance of delays in identification and transfer, high risk pregnancy identification and timely referral, capacity building of peripheral health workers in use of medication and uterine massage therapy will go a long way in reducing the maternal mortality related to atonic postpartum hemorrhage. Every pregnancy should culminate in healthy mother and healthy baby and for that we need to ensure that all women have access to high quality essential and emergency obstetric service at first referral unit (FRU) level to reduce maternal mortality.

\section{Authors' Contributions}

Dr. Mahendra.G, Dr Ramesh Babu, Dr Anshu kumari and Dr. Ravindra S Pukalecarried out the study. Dr. AnshuKumari developed the theory and performed the computations. Dr. Mahendra. G verified the analytical methods. Dr. Mahendra. G encouraged Dr. Anshu Kumari to investigate more on incidence and various methods ofmanagement of PPH in rural setup and supervised the findings of this work.
All authors discussed the results and contributed to the final manuscript.

Funding: Nil, Conflict of interest: Nil Permission from IRB: Yes

\section{References}

1. Devi KP, Singh LR, Singh LB, Singh MR and Singh NN. Postpartum haemorrhage and maternal deaths in north east India. J Obstet Gynaecol. 2015;5:635-8 http://www.scrip.org/ournal/ojog http://dx.doi.org/ 10 . 4236/ ojog. 2015. 511089

2. WHO. A newsletter of world wide activity. Making Pregnancy Safer. 2007;4:1-8.

3. Muhammad S, Chandraharan E. Chapter 23 postpartum haemorrhage, Aria's practical guide to high risk pregnancy and delivery, a south Asian perspective, Elsevier publication, 4th ed;389-98.

4. Shaikh S, Bano N, Talpur S, Balouch R. Postpartum Hemorrhage: An Experience at Tertiary Care Hospital, Hyderabad. MC. 2013;19(1):44-7.

5. Sheikh L, Najmi N, Khalid U, Saleem T. Evaluation of compliance and outcomes of a management protocol for massive postpartum hemorrhage at a tertiary care hospital in Pakistan. BMC Pregnancy Childbirth. 2011;11(1):28,1-7.

6. André Lalonde FIGO Safe Motherhood and Newborn Health (SMNH) Committee. Prevention and treatment of postpartum hemorrhage in low-resource settings. International Journal of Gynecology and Obstetrics 117 (2012) 108-118 doi:10.1016/j.ijgo.2012.03.001

7. World Health Organization. The Prevention and Management of Postpartum Haemorrhage. Report of a Technical Working Group, Geneva, 3-6 July, 1989. Unpublished document. WHO/ MCH/90.7. Geneva: World Health Organization, 1990 


\section{Original Research Article}

8. Rath W, Hackethal A, Bohlmann MK. Second-line treatment of postpartum haemorrhage (PPH). Arch Gynecol Obstet. 2012 Sep; 286(3):549-61. doi: 10.1007/s00404- 012-2329-z. Epub 2012 May 4.

9. Mousa HA, Blum J, Abou El Senoun G, et al. Treatment for primary postpartum haemorrhage. Cochrane Database Syst Rev. 2014 Feb 13;(2): CD003249. doi: 10.1002/14651858. CD003249. pub3.

10. Sheikh L, Najmi N, Khalid U, et al. Evaluation of compliance and outcomes of a management protocol for massive postpartum hemorrhage at a tertiary care hospital in Pakistan. BMC Pregnancy Childbirth. 2011 Apr 13;11:28. doi: 10.1186/1471-2393-11-28.

11. Krishna H, Chava M, Jasmine N, Shetty N: Patients with postpartum hemorrhage admitted in intensive care unit: Patient condition interventions, and outcome. J Anaesthesiol Clin Pharmacol 2011, 27(2):192-4.doi10. 4103/0970-9185.81826.

12. Condous GS, Arulkumaran S. Medical and conservative surgical management of postpartum hemorrhage. J Obstet Gynaecol Can. 2003 Nov;25(11): 931-6.

13. Csorba R. Management of post partumhaemorrhage. Orv Hetil. 2012 Apr 29;153 (17): 643-8. doi: 10.1556 /OH. 2012.29330.

14. Rath W, Hackethal A, Bohlmann MK. Second-line treatment of postpartum haemorrhage (PPH). Arch Gynecol Obstet. 2012 Sep;286(3):549-61. doi: 10.1007/s00404-012-2329-z. Epub 2012 May 4.

15. Management of postpartum hemorrhage. http://www.drplace.com/ Management_of_postpartum _ hemorrhage. 16.22850.htm.

16. Prendiville W, Elbourne D, McDonald S. Active versus expectant management in the third stage of labour. Cochrane Database Syst Rev
2009(3):CD000007. doi:10.1002/14651858. CD000007. pub2.

17. Prendiville WJ, Harding JE, Elbourne DR, Stirrat GM. The Bristol third stage trial: active versus physiological management of third stage of labour. BMJ 1988;297(6659):1295-300. PMID:3144366

18. Rogers J, Wood J, McCandlish R, et al. Active versus expectant management of third stage of labour: the Hinchingbrooke randomised controlled trial. Lancet. 1998 Mar 7;351(9104):693-9. DOI:10.1016 /S0140-6736(97)09409-9

19. United Nations. United Nations Millennium Development Goals 2000. http://www.un. org/millenniumgoals. Accessed September 15, 2011.

20. Tasneem F, Sirsam S, Shanbhag V. Clinical study of post partumhaemorrhage from a teaching hospital in Maharashtra, India. Int $\mathrm{J}$ Reprod Contracept ObstetGynecol2017 Jun;6(6):2366-2369.

21.Pranita Solanke, Swapnali Patil, Prithviraj Patil. Study of Postpartum haemorrhage in tertiary care centre. International Journal of Scientific and Research Publications, Volume 4, Issue 10, October 20141 ISSN 2250-3153 www.ijsrp.org

22. Calvert C, Thomas SL, Ronsmans C, et al. Identifying regional variation in the prevalence of postpartum haemorrhage: a systematic review and metaanalysis. PLoS One. 2012;7(7):e41114. doi: 10.1371/journal.pone.0041114. Epub 2012 Jul 23.

23. Sabrina D, Craigo, Peter S, Kapernick. Postpartum hemorrhage and abnormal puerperium. Current Obstetrics and Gynecology logic diagram. $6^{\text {th }}$ ed. London: Appleton and Lange; 1987.p 574-82.

24. Japaraj RP, Raman S. Sengstaken-Blakemore tube to control massive postpartum haemorrhage. Med J Malaysia. 2003 Oct;58 (4):604-7.

\section{How to cite this article?}

Mahendra G., Ramesh Babu, Anshu Kumari, Pukale R.S. Postpartum haemorrhage: various method of management in arural tertiary care hospital. Obs Rev: J obstet Gynecol 2019;5(2):93-98.doi:10.17511/joog.2019.i02.02. 